\title{
Minimum Cost Fault Tolerant Adder Circuits in Reversible Logic Synthesis
}

\author{
Sajib Kumar Mitra \\ Department of Computer Science and Engineering \\ University of Dhaka \\ Dhaka-1000, Bangladesh \\ Email: sajibmitra.csedu@yahoo.com
}

\author{
Ahsan Raja Chowdhury \\ Faculty of Engineering and Technology \\ University of Dhaka \\ Dhaka-1000, Bangladesh \\ Email: farhan717@univdhaka.edu
}

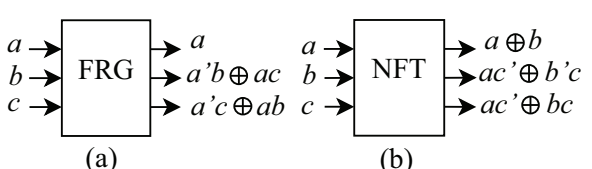

(tract-Conventional circuit dissipates energy to reload missing information because of overlapped mapping between input and output vectors. Reversibility recovers energy loss and prevents bit error by including Fault Tolerant mechanism. Reversible Computing is gaining the popularity of various fields such as Quantum Computing, DNA Informatics and CMOS Technology etc. In this paper, we have proposed the fault tolerant design of Reversible Full Adder (RFT-FA) with minimum quantum cost. Also we have proposed the cost effective design of Carry Skip Adder (CSA) and Carry Look-Ahead Adder (CLA) circuits by using proposed fault tolerant full adder circuit. The regular structures of $\boldsymbol{n}$-bit Reversible Fault Tolerant Carry Skip Adder (RFT-CSA) and Carry Look-ahead Adder (RFT-CLA) by composing several theorems. Proposed designs have been populated by merging the minimization of total gates, garbage outputs, quantum cost and critical path delay criterion and comparing with exiting designs.

Index Terms-Reversible Logic, Fault Tolerant, Carry Skip Adder, Full Adder, Quantum Cost

\section{INTRODUCTION}

Higher level of integration and use of fabrication processes have dramatically reduced the heat loss over the last decades. Landauer [1] proved that logic computation that are not reversible, necessarily generate $k T^{*} \log 2$ joules energy per bit information loss, where $k$ means Boltzman's constant and $T$ is the absolute root temperature where computation is performed. Reversible circuit doesn't loss information by considering a unique mapping between input and output. By using reversible computation zero power dissipation circuits is possible [2]. Reversible circuits are fundamentally different from traditional irreversible and are used to emphasis future technology. However reversible computation admits to generate multiple functions simultaneously. Quantum Computation is also gaining popularity as some exponentially hard problem can be solved in polynomial time and reversibility can be used to construct Quantum circuits [3].

Different arithmetic operations were realized by using reversible primitives since few decades earlier. Existing designs of Full Adder circuit were proposed in [4], [5], [6], [7] and finally generalized by [3] but any of these designs has no fault detection capability. Fault Tolerant full adder circuit was proposed in [8], [9], [10] without any generalization or cost effective structure. We have achieved more compact design of $n$-bit adder circuit which shows better performance than existing designs [6], [7], [8], [9], [12], [11]. Here we have pictured the regular structure of Fault Tolerant adder circuits by using Fault Tolerant gates (Fig. 1 shows the design of Fredkin (FRG) [13] and New Fault Tolerant (NFT) [14] gates). Rest of the paper is organized as follows: Section II discusses the construction of Reversible Logic, Fault Tolerant method, Quantum realization and Arithmetic Full Adder circuit. Section III illustrates the proposed cost effective design of Reversible Fault Tolerant Full Adder (RFT-FA), Carry Skip Adder (RFT-CSA) and Carry Look-Ahead Adder (RFT-CLA) by attaching the comparison with existing designs. Section IV describes a brief overview of the performance of proposed designs. Section V ends the paper with concluding remarks.

\section{BACKGROUND STUDY}

In this section, we have discussed about the basic definitions and properties of Reversible Logic, Fault Tolerant mechanism and Quantum realization of reversible circuit.

\section{A. Reversible Logic}

Reversible Logic always retrains an unique mapping between input and corresponding output vectors.

Definition 1. The unit logic entity of reversible circuit is called Reversible Gate where the number of inputs is equal to the number of outputs and there is an one to one mapping between input and output vectors [3].

Let, the input vector, $I_{v}=\left\{I_{1}, I_{2}, \ldots, I_{n}\right\}$ and output vector, $O_{v}=\left\{O_{1}, O_{2}, \ldots, O_{n}\right\}$ then according to the above definition the relationship is $I_{v} \leftrightarrow O_{v}$.

Definition 2. The input vector, $I_{v}$ and output vector, $O_{v}$ for $2 \times 2$ Feynmen Gate (FG) [15] is defined as follows:

$$
\begin{aligned}
I_{v} & =\{a, b\} \text { and } \\
O_{v} & =\{a, a \oplus b\}
\end{aligned}
$$




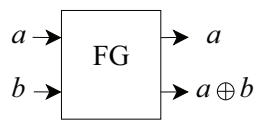

Fig. 2. $2 \times 2$ Feynman/CNOT Gate

TABLE I

Truth TABle of Feynman Gate

\begin{tabular}{|c|c|c|c|}
\hline \multicolumn{2}{|c|}{ Input } & \multicolumn{2}{c|}{ Output } \\
\hline$a$ & $b$ & $a$ & $a \oplus b$ \\
\hline 0 & 0 & 0 & 0 \\
\hline 0 & 1 & 0 & 1 \\
\hline 1 & 0 & 1 & 1 \\
\hline 1 & 1 & 1 & 0 \\
\hline
\end{tabular}

Fig. 2 shows the block diagram of Feynman Gate and Table I shows the unique mapping between input and output vectors of Feynman gate.

Definition 3. The Garbage Output of any reversible gate or circuit is unwanted or unused output which will not be used in future rather than for checking reversibility [3].

For example, the Exclusive-OR operation can be realized by using only one Feynman Gate which produces an extra dummy output $(a)$ along with its principle output signal $(a \oplus$ b) to preserve reversibility (shown in Fig. 2).

Definition 4. Delay of any circuit is the number of maximum gate(s) from any input to any output where both ends preserve a continuous communication line. Total delay to generate EX-OR function is 1 (shown in Fig. 2) [3].

Definition 5. The input vector, $I_{v}$ and output vector, $O_{v}$ of $3 \times 3$ Fredkin Gate (FRG) [13] is defined as follows:

$$
\begin{aligned}
I_{v} & =\{a, b, c\} \text { and } \\
O_{v} & =\{a, \bar{a} b \oplus a c, \bar{a} c \oplus a b\}
\end{aligned}
$$

The block representation of FRG is shown in Fig. 1(a).

Definition 6. The $3 \times 3$ dimensional Feynman Double Gate (F2G) [16] is another reversible gate where the input vector, $I_{v}$ and the output vector, $O_{v}$ are defined as follows:

$$
\begin{aligned}
I_{v} & =\{a, b, c\} \text { and } \\
O_{v} & =\{a, a \oplus b, a \oplus c\}
\end{aligned}
$$

The Block Diagram and the unique mapping between input and output vectors of Feynman Double (F2G) gate can be shown as Fig. 3 and Fig. 4 respectively.

Definition 7. The input vector, $I_{v}$ and output vector, $O_{v}$ of $3 \times 3$ New Fault Tolerant (NFT) gate [14] as follows:

$$
\begin{aligned}
& I_{v}=\{a, b, c\} \text { and } \\
& O_{v}=\{a \oplus b, a \bar{c} \oplus \bar{b} c, a \bar{c} \oplus b c\}
\end{aligned}
$$

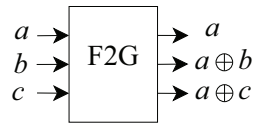

Fig. 3. $3 \times 3$ Feynman Double Gate

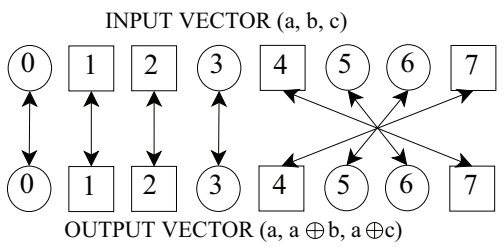

Fig. 4. Feynman Double Gate (F2G) preserves Fault Tolerance over inputoutput unique mapping

The block diagram of NFT gate is shown in Fig. 1(b).

Definition 8. The input and output vectors of $4 \times 4$ Modified TSG (MTSG) gate [3] are $I_{v}$ and $O_{v}$ respectively and can be defined as follows:

$$
\begin{aligned}
I_{v} & =\{a, b, c, d\} \text { and } \\
O_{v} & =\{a, a \oplus b, a b \oplus c,(a \oplus b) c \oplus a b \oplus d\}
\end{aligned}
$$

Another popular reversible gates are Peres Gate (PG) [18], Toffoli Gate (TG) [17] and New Gate (NG) [19] etc.

\section{B. Fault Tolerant Method}

Reversibility recovers bit loss but is not able to detect bit error in circuit. Fault Tolerant reversible circuit is capable to prevent error at outputs.

Definition 9. Fault Tolerant (FT) gate, also called Conservative Reversible Gate [9] which means the Hamming weight of its input and output are equal.

Let, the input and output vectors of any Fault Tolerant gate are $I_{v}=\left\{I_{0}, I_{1}, \ldots, I_{n-1}\right\}$ and $O_{v}=\left\{O_{0}, O_{1}, \ldots, O_{n-1}\right\}$ where the following equations (1) and (2) must be preserved:

$$
\begin{aligned}
I_{v} & \leftrightarrow O_{v} \\
I_{0} \oplus I_{1} \oplus \ldots \oplus I_{n-1} & =O_{0} \oplus O_{1} \oplus \ldots \oplus O_{n-1}
\end{aligned}
$$

For example, the Fault Tolerance property of Feynman Double gate (shown in Fig. 3) can be verified from Fig. 4 where square and circle represents ODD and EVEN parities respectively and the equivalent decimal values of input-output vectors are represented as corresponding decimal number (0-7).

F2G, FRG and NFT are $3 \times 3$ dimensional and MIG is $4 \times 4$ dimensional fault tolerant gate having unique mapping between Input and Output vectors. The input and corresponding output parities of Fault Tolerant gates are same [16]. In early, fault tolerant Gate is also called Parity Preserving Gate.

\section{Quantum Realization}

Quantum realization is another fact to judge the efficiency of reversible circuit which uses matrix multiplication rather than conventional Boolean operations. In Quantum Mechanics, the states of a particle is represented by qubits instead of bits. The operations over on qubits are matrix multiplication specified by using quantum gates (shown in Fig. 5) [20].

Definition 10. Quantum Cost (QC) of any reversible circuit is the total number of $2 \times 2$ quantum primitives that are used to realize equivalent quantum circuit [3], [21]. 


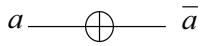

(a)

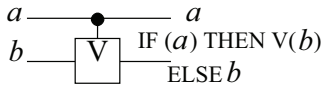

(c)

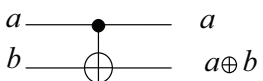

(b)

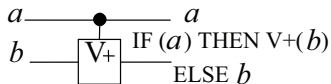

(d)
Fig. 5. Elementary Quantum Logic gates: (a) NOT, (b) Exclusive-OR, (c) Square Root of NOT (SRN) and (d) Hermitian matrix of SRN

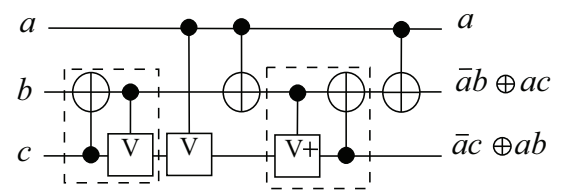

Fig. 6. Quantum circuit realization of Fredkin gate [13]

For example, the quantum cost of Feynman gate (shown in Fig. 2) is one because single $2 \times 2$ Quantum EX-OR gate is enough to realize its operations. The quantum circuit of several reversible gates is presented in [3]. Fig. 6 shows the quantum representation of Fredkin gate and the cost is 5 .

Here we have proposed the quantum realization of reversible fault tolerant Modified IG (MIG) [8] and New Fault Tolerant (NFT) [14] gates by using quantum EX-OR, Square Root of NOT (SRN or V) and Hermitian of SRN $\left(\mathrm{V}^{+}\right)$gates as shown in Fig. 7. According to design, the quantum cost of Modified IG (MIG) (New Fault Tolerant (NFT)) gate is 7(5).

\section{Arithmetic Adder Circuit}

Adder is an essential part of digital circuits to implement most of the mathematical operations. Single bit adder or Full Adder is the unit entity of any kind of computing devices.

Definition 11. A Full Adder is a digital circuit which takes two bits from two operands and carry bit from prior stage as input and generates the summation of three bits and corresponding carry as output [3].

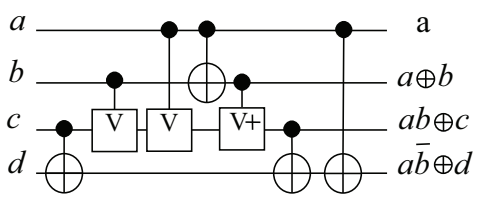

(a) Modified IG gate, Quantum Cost $=7$

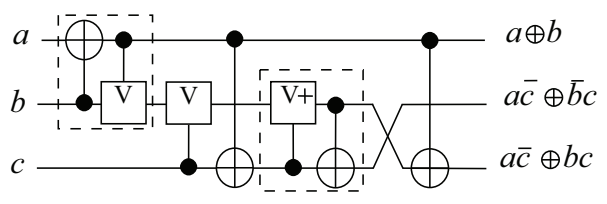

(b) New Fault Tolerant Gate, Quantum Cost $=5$

Fig. 7. Quantum equivalent circuit realization of MIG [8] and NFT [14] by using quantum primitives

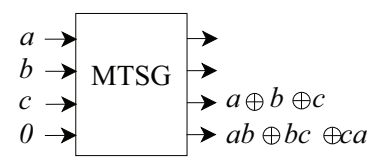

Fig. 8. Full adder Realization by using MTSG [3]

Let, $a$ and $b$ both are the single bit operand and $c_{i n}$ is the carry of previous stage then outputs of full adder circuit, Sum $(s)$ and Carry $\left(c_{\text {out }}\right)$ can be defined as follows:

$$
\begin{aligned}
s & =a \oplus b \oplus c_{i n} \\
c_{\text {out }} & =a c_{\text {in }} \oplus b c_{\text {in }} \oplus a b
\end{aligned}
$$

For example, single MTSG gate able to realize reversible full adder circuit as shown in Fig. 8.

Following section has described the proposed designs of Fault Tolerant Full Adder (RFT-FA) circuit followed by Carry Skip Adder (RFT-CSA) and Carry Look-ahead Adder (RFTCLA) by including their performances over existing designs.

\section{PROPOSED DESIGN}

In this section, first we have described the proposed design of cost effective Reversible Fault Tolerant Full Adder by using New Fault Tolerant (NFT) and Feynman Double (F2G) gates. Then we have described the design of Fault Tolerant Carry Skip (RFT-CSA) and Carry Look-ahead (RFT-CLA) adders by using proposed design of Fault Tolerant Full Adder.

\section{A. Fault Tolerant Full Adder Design}

The quantum cost of New Fault Tolerant (NFT)(shown in Fig. 7(b)) and Fredkin (FRG) gates are same i.e. 5. But the quantum cost of Feynman Double (F2G) gate is 2. We have used New Fault Tolerant (NFT) and Feynman Double (F2G) gates because of reusability of proposed adder circuit for Carry Skip Adder and Carry Look-ahead Adder.

Definition 12. Single NFT Full Adder (SNFA) is a Fault Tolerant full adder circuit which consists of one New Fault Tolerant (NFT) gate and three Feynman Double (F2G) gates where the quantum cost is 11 and the total number of garbage output is 3 (shown in Fig. 9).

Theorem 1: The minimum number of garbage bit to realize Reversible Fault Tolerant Full Adder circuit is 3.

Proof: Let, $a, b$ and $c_{i n}$ are the inputs of a full adder circuit where $s$ and $c_{\text {out }}$ are the corresponding outputs. There are three different states at the inputs $\left(a, b\right.$ and $\left.c_{i n}\right)$ where the outputs ( $s$ and $c_{\text {out }}$ ) produce same patterns as shown in Table II. For any parity preserving reversible circuit, total number of EVEN or ODD parity at input or output is equal. Table II shows that the all input patterns are EVEN but the corresponding output patterns are ODD. Turning three ODD patterns at output into EVEN by adding two extra bits is not possible. Because two bits can represent $2^{2}$ different states where 00 and 11 (01 and 10) are EVEN (ODD) only. So, Reversible Full Adder circuit requires at least 3 garbage bits to make itself Reversible Fault Tolerant Full Adder. 
TABLE II

INPUT-OUTPUT PATTERNS OF FULL ADDER

\begin{tabular}{|c|c|c|c|c|}
\hline \multicolumn{3}{|c|}{ Input } & \multicolumn{2}{c|}{ Output } \\
\hline$a$ & $b$ & $c_{\text {in }}$ & $s$ & $c_{\text {out }}$ \\
\hline 0 & 1 & 1 & 0 & 1 \\
\hline 1 & 1 & 0 & 0 & 1 \\
\hline 1 & 0 & 1 & 0 & 1 \\
\hline
\end{tabular}

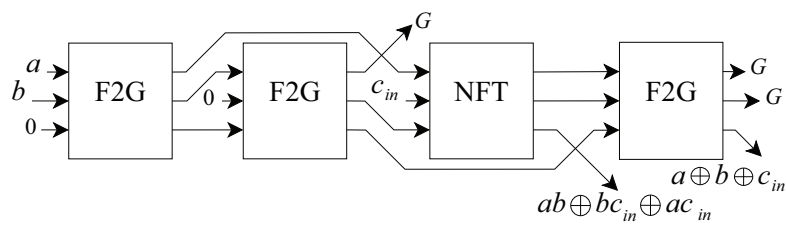

Fig. 9. Proposed design of Fault Tolerant Single NFT Full Adder (SNFA)

TABLE III

COMPARISON BETWEEN PROPOSED AND EXISTING DESIGNS OF REVERSIBLE FAULT TOLERANT FULL ADDER

\begin{tabular}{|c|c|c|c|c|}
\hline Fault Tolerant & \multicolumn{2}{|c|}{ Total Gates } & \multirow{2}{*}{ GB } & \multirow{2}{*}{ QC } \\
\cline { 2 - 3 } Full Adder & $3 \times 3$ & $4 \times 4$ & & \\
\hline Proposed & 4 & 0 & 3 & 11 \\
\hline Existing [8] & 0 & 2 & 3 & 14 \\
\hline Existing [9] & 5 & 0 & 4 & 25 \\
\hline Existing [10] & 6 & 0 & 6 & 18 \\
\hline
\end{tabular}

$\mathrm{GB}=$ Garbage bits, $\mathrm{QC}=$ Quantum Cost

The performance analysis between the proposed design and all other existing designs is shown in Table III.

Table III shows that the Ref. [8] uses two gates and the corresponding quantum cost is 14 where proposed design uses low dimensional four gates and the quantum cost is only 11.

\section{B. Fault Tolerant Carry Skip Adder}

Fast carry emission is the main concern of Carry Skip Adder and it depends on: firstly, if any operand is equal to logical 1 then the full adder propagates $c_{i n}$ to $c_{o u t}$ and secondly, it also generates carry itself ( $\mathrm{c}_{\text {out }}$ independent on $\mathrm{c}_{i n}$ ).

Definition 13. Propagate is a simple XOR operation between two operands which is responsible for only bypassing the carry of previous stage to next stage [6].

Let, $X=\left(x_{0}, x_{1}, x_{2}, \ldots, x_{n-1}\right)$ and $Y=\left(y_{0}, y_{1}, y_{2}, \ldots, y_{n-1}\right)$ are two $n$-bit operands where Propagate $p_{i}$ of $i^{\text {th }}$ stage can be defined from $x_{i}$ and $y_{i}$ as follows:

$$
p_{i}=x_{i} \oplus y_{i}
$$

Definition 14. Generate is an AND operation which enables current stage of adder to generate carry for next stage [6]. So the Generate of $i^{t h}$ stage as follows:

$$
g_{i}=x_{i} y_{i}
$$

Definition 15. Reversible Fault Tolerant Carry Skip Adder (RFT-CSA) consists of SNFAs and FRGs to perform summation and propagate carry respectively which reduce the delay or bypassing carry due to the recalculation of carry for the next stage. If any input is equal to a logical 1 , then it propagates the carry input to the carry output [9].
Proposition 1. $n$-bit RFT-CSA can be realized by using $(3 n+1)$ F2Gs, $n$ FRGs and $n$ NFTs.

Proof: Let, $n$ SNFAs are needed to realize $n$-bit RFT-CSA (each SNFA consists of three F2Gs and one NFT) to generate sum $\left(s_{i}\right)$ and Propagate $\left(p_{i}\right)$ where $i=0,1,2, \ldots,(n-1)$. And $n$ FRGs are needed for performing AND operation among $n$ Propagates with $c_{i n}$. So the calculation of the number of NFT $\left(N F T_{C S A}\right)$, the number of FRG $\left(F R G_{C S A}\right)$ and the number of F2G $\left(F 2 G_{C S A}\right)$ to implement $n$-bit RFT-CSA is as follows:

$$
\begin{aligned}
N F T_{C S A} & =n, \\
F R G_{C S A} & =n \text { and } \\
F 2 G_{C S A} & =3 n
\end{aligned}
$$

But RFT-CSA needs another extra F2G to generate final carry, $c_{\text {out }}$ by performing EXOR operation between $c_{n-1}$ and $\left(p_{n-1} p_{n-2} \ldots p_{0} \mathrm{c}_{i n}\right)$.

$$
F 2 G_{C S A}=3 n+1
$$

So, $n$-bit RFT-CSA can be realized by using $(3 n+1)$ F2Gs, $n$ FRGs and $n$ NFTs.

Proposition 2. $n$-bit RFT-CSA can be realized with $(n+5)$ Critical Path Delay.

The proposed design of 4-bit RFT-CSA is shown in Fig. 10 which uses proposed full adder (SNFA) circuit.

Finally, the total garbage $\left(G B_{C S A}\right)$ and Quantum Cost $\left(Q C_{C S A}\right)$ of $n$-bit RFT-CSA can be written as follows:

$$
\begin{aligned}
G B_{C S A} & =4 n \\
Q C_{C S A} & =5 * 2 n+2 *(3 n+1) \\
& =16 n+2
\end{aligned}
$$

The comparison between proposed RFT-CSA and existing designs is shown in Table IV. Carry Skip Adder is more reliable in case of hardware implementation where circuit cost is another factor of design with respect to Delay. In Table IV, the QC of proposed design is 66 which is minimum than all existing designs. Although the number of $3 \times 3$ gates of [7] is about equal to proposed design but QC of proposed design has been improved $20 \%$ because of using cost effective Feynman Double gate (QC of Feynman Double gate is only 2). The proposed cost-effective design of Fault Tolerant CSA (RFTCSA) has improved cost factor having fault detection as well.

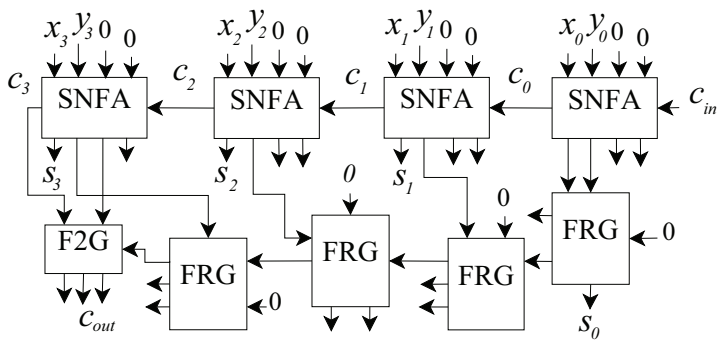

Fig. 10. Proposed design of Reversible Fault Tolerant Carry Skip Adder 
TABLE IV

COMPARISON BETWEEN PROPOSED AND EXISTING DESIGNS OF CARRY SKIP ADDER

\begin{tabular}{|c|c|c|c|c|c|}
\hline \multirow{2}{*}{$\begin{array}{c}\text { Carry Skip } \\
\text { Adder }\end{array}$} & \multicolumn{2}{|c|}{ Total Gates } & \multirow{2}{*}{ GB } & \multirow{2}{*}{ DL } & \multirow{2}{*}{ QC } \\
\hline & $3 \times 3$ & $4 \times 4$ & & & \\
\hline Proposed $\dagger$ & 21 & 0 & 16 & 9 & 66 \\
\hline Existing [7] $\S$ & 22 & 0 & 26 & 16 & 88 \\
\hline Existing [8]† & 6 & 8 & 19 & 13 & 80 \\
\hline Existing [9]§ & 24 & 0 & 23 & 14 & 120 \\
\hline
\end{tabular}

\section{Fault Tolerant Carry Look-ahead Adder Circuit}

This section introduces the design of Reversible Fault Tolerant Carry Look-ahead Adder (RFT-CLA) circuit overlaps the performance of all existing designs. Proposed design of RFTCLA is based on New Fault Tolerant (NFT) and Feynman Double $(\mathrm{F} 2 \mathrm{G})$ gates where the carry is generated before sum.

Definition 16. Reversible Fault Tolerant Carry Lookahead Adder (RFT-CLA) consists of serial attachment of $n$ SNFAs but the work as a carry generator itself where the carry output of $i^{t h}$ stage $\left(c_{i}\right)$ is produced before sum $s_{i}$ where $i=0$, $1,2, \ldots,(n-1)$.

Proposition 3. $n$-bit RFT-CLA can be realized by using the combination of $n$ NFTs and $n$ F2Gs.

Proposition 4. The Delay of $n$-bit Reversible Fault Tolerant CLA $\left(D_{R F T-C L A}\right)$ can be minimized to $(n+3)$.

Proof: According to Definition 4, the Delay of any circuit is the number of maximum gates laying on contiguous path of any input to output. The Delay of SNFA, $D_{S N F A}=4$ to generate sum not carry. Delay of parallel adder circuit depends on carry propagation (from $c_{i n}$ to $c_{\text {out }}$ ) of every stage. Any $n$-bits RFT-CLA needs $n$ SNFAs where Delay of RFTCLA, $D_{R F T-F A} \neq 4 n$. Because carry input $\left(c_{i}\right)$ of $i_{t h}$ stage is generated by spending 1 units Delay where $i=0,1,2, \ldots$, $(n-1)$. In first stage, extra two units Delay is added because

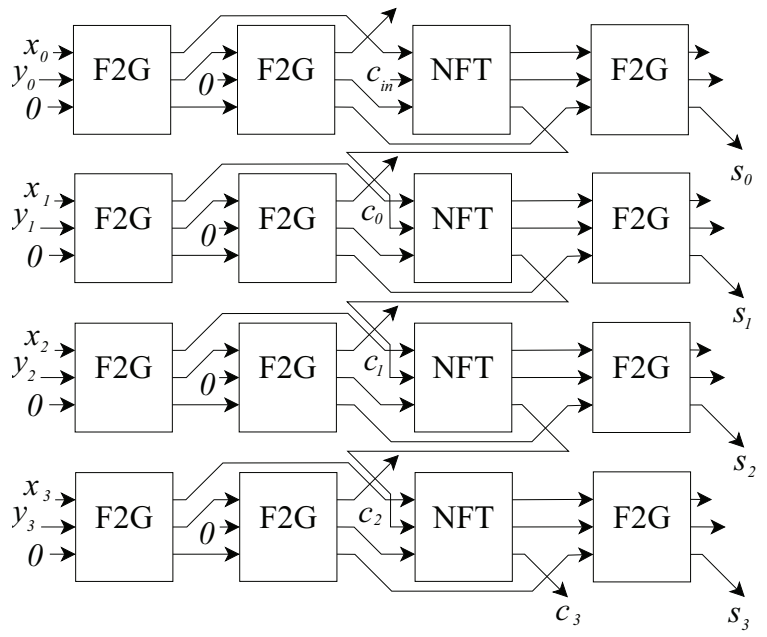

Fig. 11. Efficient Design of 4-bit Reversible Fault Tolerant Carry Look-ahead Adder
TABLE V

COMPARISON BETWEEN PROPOSED AND EXISTING DESIGN [8] OF FAULT TOLERANT CARRY LOOK-AHEAD ADDER

\begin{tabular}{|c|c|c|c|c|c|}
\hline Fault Tolerant & \multicolumn{2}{|c|}{ Total Gates } & \multirow{2}{*}{ GB } & \multirow{2}{*}{ DL } & \multirow{2}{*}{ QC } \\
\cline { 2 - 3 } Carry Look-ahead Adder & $3 \times 3$ & $4 \times 4$ & & & \\
\hline Proposed (4-bit) & 16 & 0 & 12 & 7 & 44 \\
\hline Existing [8] (2-bit) & 15 & 4 & 28 & 12 & 73 \\
\hline
\end{tabular}

of first carry output $\left(c_{0}\right)$ generation is related to operands at first stage. On the other hand, last stage has extra single unit Delay because the final sum is generated after one stage of generation of final carry $\left(c_{\text {out }}\right)$. So the Delay calculation for $n$-bits RFT-CLA is as follows:

$$
D_{R F T-F A}=n+3
$$

Therefore an $n$-bit RFT-CLA can be realized by using $(n+3)$ unit Delay.

Proposition 5. An n-bit Reversible Fault Tolerant Carry Look-ahead Adder (RFT-CLA) can be realized with minimum Quantum Cost $11 n$.

Proposition 6. An $n$-bit Reversible Fault Tolerant Carry Look-ahead Adder (RFT-CLA) can be realized with minimum Garbage 3n.

Fig. 11 shows the proposed design of 4-bit Reversible Fault Tolerant Fast Adder and Table V shows the performance of proposed design by comparing with existing [8] design of 2-bit reversible Fault Tolerant Carry Look-ahead Adder.

\section{Performance Analysis}

Previous two sections have discussed about the design of Reversible Fault Tolerant Full Adder, Carry Skip Adder and Carry Look-ahead Adder and the comparison of corresponding existing designs. This section have presented an abstract overview of proposed designs and the complexity analysis for $n$-bit Reversible Fault Tolerant Adder circuits. Fig. 12 represents that the performance of proposed 4-bit Reversible Fault Tolerant Carry Skip Adder is better compared to existing [8]. The number of gates in proposed design is greater than existing design [8] because of dimensional impact (lower dimension is preferable) which can be treated as negligible because of other factors (delay, garbage and quantum cost). Table VI describes the another evolutionary observation between proposed $n$-bit Reversible Fault Tolerant Carry Skip Adder and Carry Lookahead Adder designs. We have already given the comparative study between proposed and existing designs of reversible fault tolerant Carry Skip Adder (Carry Look-ahead Adder) in Table IV (Table V) individually. Proposed designs of CSA and CLA demand better performance than existing designs in terms of number of gates, garbage outputs, delay and quantum cost. Along with the lower dimensional $(3 \times 3)$ fault tolerant gates our proposed designs have got more flexibility in reversible CMOS [22] realization. Pictorial representation of performance evaluation of Reversible Fault Tolerant Carry Look-ahead adder circuit over Carry Skip Adder circuit is shown in Fig. 13. 


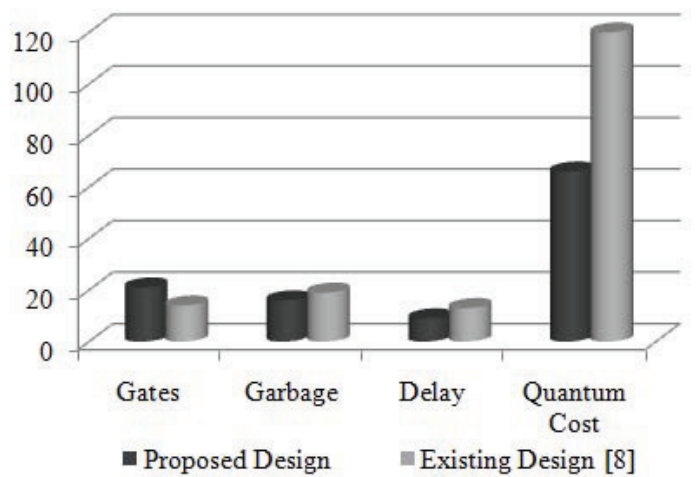

Fig. 12. Comparison between Proposed design and Existing design [8] of 4-bit Carry Skip Adder

TABLE VI

COMPARISON AMONG PROPOSED REVERSible FAUlt TOLERANT FAST, CARRY SKIP AND CARRY LOOK AHEAD ADDER

\begin{tabular}{|c|c|c|c|c|c|}
\hline $\begin{array}{c}\text { Proposed } \\
\text { Adder Cirucits }\end{array}$ & \multicolumn{2}{|c|}{ Total Gates } & \multirow{2}{*}{ GB } & \multirow{2}{*}{ DL } & \multirow{2}{*}{ QC } \\
\cline { 2 - 4 } & FRG & F2G & & & \\
\hline RFT-CLA $\dagger$ & $n$ & $3 n$ & $3 n$ & $n+3$ & $11 n$ \\
\hline RFT-CSA & $2 n$ & $3 n+1$ & $4 n$ & $n+5$ & $16 n+2$ \\
\hline
\end{tabular}

$\mathrm{GB}=$ Garbage bits, $\mathrm{DL}=$ Delay, $\mathrm{QC}=$ Quantum cost

\section{CONCLUSION}

In our proposed designs, we have combined all marginal cost factors (Gate cost, Delay, Garbage and Quantum cost) to generate optimized architecture of Reversible Fault Tolerant adder circuits which gather better performance than existing all fault tolerant designs. This paper has covered the designs of minimum cost fault tolerant Carry Skip Adder (RFT-CSA) and Carry Look-ahead Adder Circuits. Both designs have used the proposed structure of fault tolerant Full Adder (RFT-FA or SNFA) circuit has minimum quantum cost 11 . Several number of theorems have been proposed to make the designs of RFTCSA and RFT-CLA more generalized for $n$-bit fault tolerant adder circuitry. Finally, we have attached the evolutionary report of performance of proposed designs.

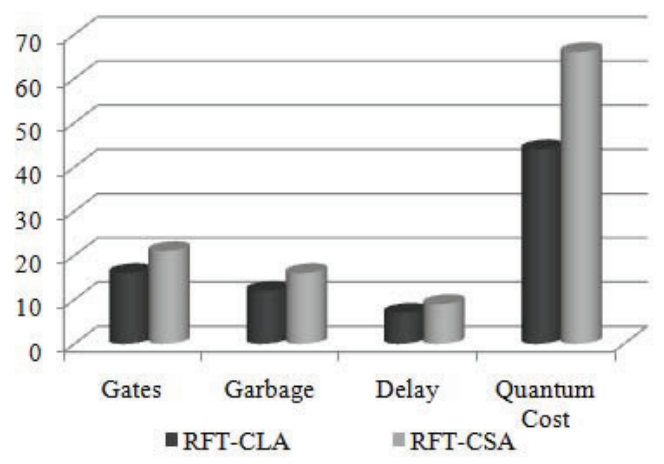

Fig. 13. Comparison between Proposed design of 4-bit Reversible Fault Tolerant Carry Skip Adder and Carry Look-ahead Adder

\section{REFERENCES}

[1] R. Landauer, "Irreversibility and heat generation in the computing process," IBM Journal Of Research and Development, vol. 5, pp. 183$191,1961$.

[2] C. H. Bennett, "Logical reversibility of computation," IBM Journal Of Research and Development, vol. 17, pp. 525-532, 1973.

[3] A. K. Biswas, M. M. Hasan, A. R. Chowdhury, and H. M. H. Babu, "Efficient approaches for designing reversible binary coded decimal adders," Microelectronics Jounrnal, vol. 39, no. 12, pp. 1693-1703, December 2008.

[4] H. M. H. Babu and A. R. Chowdhury, "Design of a compact reversible binary coded decimal adder circuit," Elsevier Journal Syst. Archit., vol. 52, no. 5, pp. 272-282, January 2006.

[5] H. M. H. Babu, M. R. Islam, A. R. Chowdhury, and S. M. A. Chowdhury, "Reversible logic synthesis for minimization of full-adder circuit," in 17th International Conference on VLSI design'04. Mumbai, India, 2004, pp. 757-760.

[6] H. Thapliyal and M. B. Srinivas, "A novel reversible tsg gate and its application for designing reversible carry look-ahead and other adder architectures," in Asia-Pacific Computer Systems Architecture Conference, 2005, pp. 805-817.

[7] P. K. Lala, J. P. Parkerson, and P. Charaborty, "Adder designs using reversible logic gates," WSEAS TRANSACTIONS on CIRCUITS and SYSTEMS, June 2010.

[8] M. S. Islam, M. M. Rahman, Z. begum, and M. Z. Hafiz, "Efficient approaches for designing fault tolerant reversible carry look-ahead and carry-skip adders," MASAUM Journal of Basic and Applied Sciences, vol. 1, no. 3, 2009.

[9] J. W. Bruce et al., "Efficient adder circuits based on a conservative reversible logic gates," in ISVLSI '02: Proceedings of the IEEE Computer Society Annual Symposium on VLSI. Washington, DC, USA, 2005, pp. 83-88.

[10] M. Haghparast and K. Navi, "Design of a novel fault tolerant reversible full adder for nanotechnology based systems," World Applied Science Journal, vol. 3, no. 1, pp. 114-118, 2008.

[11] B. Desoete and A. D. Vos, "A reversible carry-look-ahead adder using control gates," Intregation the VLSI Journal, vol. 33, no. 1, pp. 89-104, 2002.

[12] J. Lim, D. Kim, and S. Chae, "A 16-bit carry-lookahead adder using reversible energy recovery logic for ultra-low-energy systems," IEEE J. Solid-State Circuits, vol. 34, pp. 898-903, 1999.

[13] E. Fredkin and T. Toffoli, "Conservative logic," International Journal Of Theoretical Physics, vol. 21, pp. 219-253, 1982.

[14] M. Haghparast and K. Navi, "Novel fault tolerant gate for nanotechnology based systems," American Journal of Applied Sciences, vol. 5, no. 5, pp. 519-523, 2008.

[15] R. P. Feynman, "Quantum mechanical computers," Opt. News, vol. 11, no. 2, pp. 11-20, 1985 .

[16] B. Parhami, "Fault tolerant reversible circuits," in In Proc. of 40th Asimolar Conference Signals, Systems and Computers. Pacific Grove, CA, 2006, pp. 1726-1729.

[17] T. Toffoli, "Reversible computing," MIT Lab for Computer Science, vol. 85, pp. 632-644, 1980.

[18] A. Peres, "Reversible logic and quantum computers," Physics Review A., vol. 32, no. 6, pp. 3266-3276, December 1985.

[19] M. H. A. Khan and M. A. Perkowski, "Logic synthesis with cascades of new reversible gate families," in 6th International Symposiumon Representation and Methodology of Future Computing Technology (ReedMuller), March 2003, pp. 43-55.

[20] W. N. N. Hung, X. Song, G. Yang, J. Yang, and M. Perkowski, "Quantum logic synthesis by symbolic reachability analysis," in 41st Conference on (DAC'04), Design Automation Conference, May 2004, pp. 838-841.

[21] M. Perkowski and et al, "A hierarchical approach to computer-aided design of quantum circuits," in 6th International Symposium on Representations and Methodology of Future Computing Technology, 2003, pp. 201-209.

[22] Y. V. Rentergem and A. D. Vos, "Optimal design of a reversible full adder," International Journal of Unconventional Computing, vol. 1, pp. 339-355, 2005. 